\title{
Overcoming Selectivity Issues in Reversible Catalysis: A Transfer Hydrocyanation Exhibiting High Kinetic Control
}

\section{Journal Article}

Author(s):

Bhawal, Benjamin (1); Reisenbauer, Julia C.; Ehinger, Christian; Morandi, Bill

Publication date:

2020-06-24

Permanent link:

https://doi.org/10.3929/ethz-b-000424373

Rights / license:

$\underline{\text { In Copyright - Non-Commercial Use Permitted }}$

Originally published in:

Journal of the American Chemical Society 142(25), https://doi.org/10.1021/jacs.0c03184

Funding acknowledgement:

757608 - Shuttle Catalysis for Reversible Molecular Construction (EC) 
This document is the Accepted Manuscript version of a Published Work that appeared in final form in the Journal of the American Chemical Society, copyright (C) American Chemical Society after peer review and technical editing by the publisher. To access the final edited and published work see https://doi.org/10.1021/jacs.0c03184

\title{
Overcoming Selectivity Issues in Reversible Catalysis - A Transfer Hydrocyanation Exhibiting High Kinetic Control
}

\author{
Benjamin N. Bhawal, ${ }^{+, \neq}$Julia C. Reisenbauer, ${ }^{\dagger, \neq}$ Christian Ehinger, ${ }^{\dagger}$ Bill Morandi ${ }^{*,+, \neq}$ \\ †ETH Zürich, Vladimir-Prelog-Weg 3, HCI, 8093 Zürich, Switzerland \\ ${ }^{\ddagger}$ Max-Planck-Institut für Kohlenforschung, Kaiser-Wilhelm-Platz 1, 45470 Mülheim an der Ruhr, Germany
}

Supporting Information. Detailed experimental procedures and characterization data.

\begin{abstract}
Reversible catalytic reactions operate under thermodynamic control and thus establishing a selective catalytic system poses a considerable challenge. Herein, we report a reversible transfer hydrocyanation protocol that exhibits high selectivity for the thermodynamically less favorable branched isomer. Selectivity is achieved by exploiting the lower barrier for $\mathrm{C}-\mathrm{CN}$ oxidative addition and reductive elimination at benzylic positions in the absence of co-catalytic Lewis acid. Through the design of a novel type of HCN donor, a practical, branched-selective, HCN-free transfer hydrocyanation was realized. The synthetically useful resolution of a mixture of branched and linear nitrile isomers was also demonstrated to underline the value of reversible and selective transfer reactions. In a broader context, this work demonstrates that high kinetic selectivity can be achieved in reversible transfer reactions, thus opening new horizons for their synthetic applications.
\end{abstract}

Reversible catalytic reactions such as alkene metathesis ${ }^{1}$ and transfer hydrogenation ${ }^{2}$ have found a plethora of applications in diverse fields of chemistry. ${ }^{3,45}$ Given their value to the synthetic practitioner, our group and others have investigated expanding these transformations to include the metathesis of other bonds and the transfer of functionality other than $\mathrm{H}_{2}{ }^{6,7,8}$ These reaction paradigms offer numerous advantages such as implementing safer protocols avoiding the use of highly toxic reagents or unlocking previously unknown transformations. Our group applied a shuttle catalysis strategy to achieve transfer hydrocyanation whilst avoiding the use of highly toxic and volatile $\mathrm{HCN} .{ }^{8 \mathrm{~d}}$ Notably, this strategy also enabled the first example of dehydrocyanation of aliphatic nitriles. These results have subsequently spurred the development of other transfer hydrocyanation protocols that circumvent the use of $\mathrm{HCN}^{9}$ as well as opening new approaches for other cyanation reactions using relatively non-toxic cyanide sources. ${ }^{10,11}$

One fundamental drawback of metathesis and shuttle catalysis reactions is that, due to their inherent reversibility, they operate under thermodynamic control. This often leads to mixtures of products being formed such as in the case for alkene metathesis wherein the ratio of $E: Z$ products is determined by their relative stability (Scheme 1A). ${ }^{1}$ In a similar manner, the transfer hydrocyanation reported by our group also leads to mixtures of products, with the reaction of styrene affording an 81:19 mixture of linear and branched products, respectively. ${ }^{8 \mathrm{~d}}$ Again, this ratio reflects the relative thermodynamic stability of the two regioisomers. ${ }^{12}$

Scheme 1. Context of the work. 


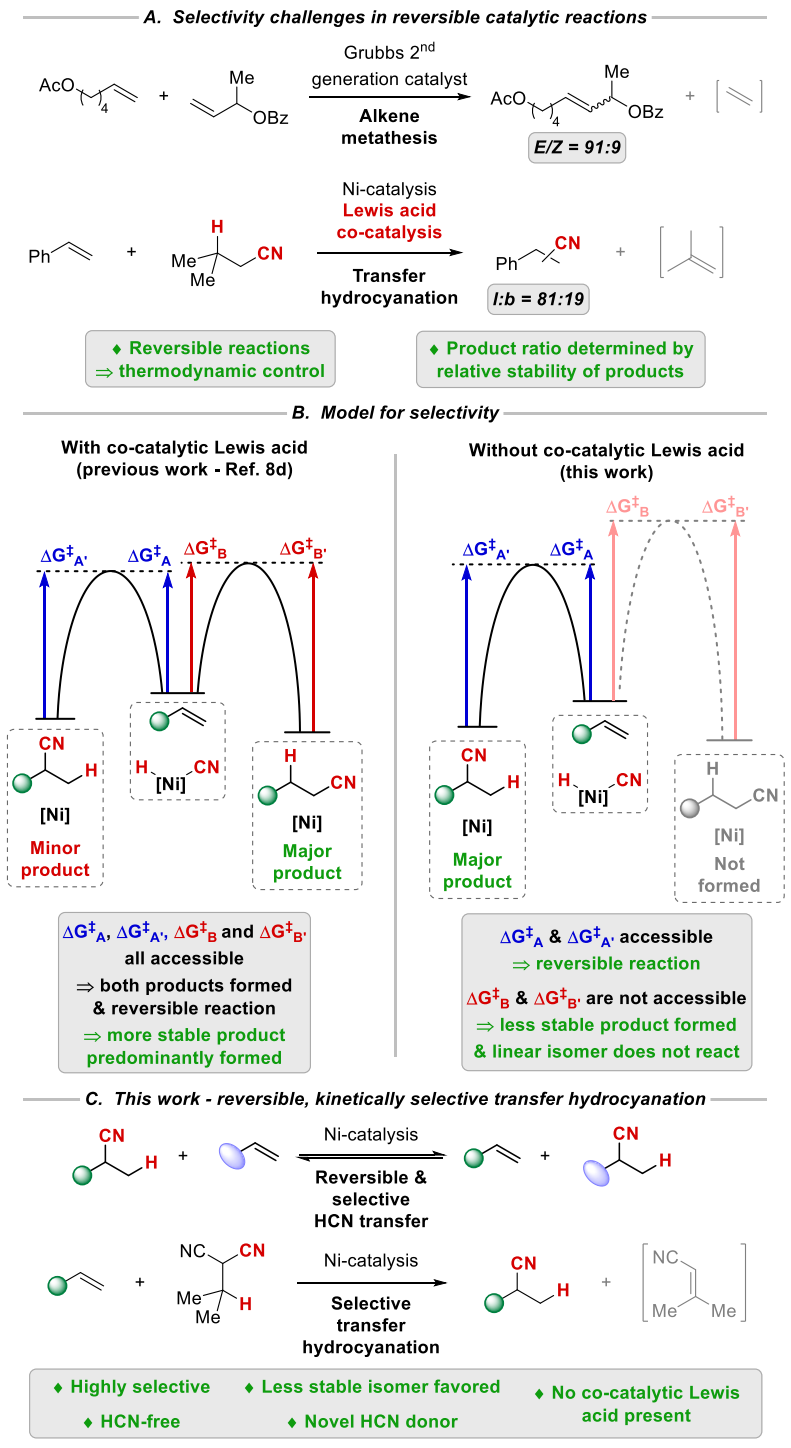

These reactions are reversible because the products possess the same functionality as the starting materials and thus are roughly ergoneutral (Scheme 1B). Hence, in most cases, the barrier for both the forward and reverse reaction is similar. In reactions where the formation of more than one isomer is kinetically accessible, the selectivity is determined by their relative stability. To override this thermodynamic bias in selectivity, the barrier for one of the isomers must be kinetically inaccessible. In this scenario, the reaction will remain reversible but restricted to a smaller subset of isomers. This is the case in $Z$-selective alkene metathesis, wherein the intricate design of the catalysts lead to a significantly higher activation barrier for the formation and reaction of $E$-alkenes. ${ }^{13}$ As a result, $Z$-alkenes are exclusively formed and, conversely, if a mixture of $Z$-and $E$-alkenes are present, then only the $Z$-alkenes will react. To date, there are no analogous examples of a transfer reaction that has been demonstrated to be reversible and kinetically selective. Instead, most reversible transfer reactions proceed to form the more thermodynamically stable products.

Inspired by the precedent set by $Z$-selective alkene metathesis to obtain a selective and reversible metathesis manifold, ${ }^{13}$ we sought to develop a protocol that would enable a selective and reversible transfer hydrocyanation of styrenes by manipulating the difference in the barrier for the formation of the branched and linear nitrile isomers to selectively form the less stable branched isomer (Scheme 1C). This would be complementary to the linear selectivity obtained in previous transfer hydrocyanation reactions. ${ }^{8 \mathrm{~d}, 9 \mathrm{a}}$ Several groups have demonstrated that co-catalytic Lewis acids help facilitate both $\mathrm{C}-\mathrm{CN}$ oxidative addition and reductive elimination, ${ }^{14,15}$ resulting in a thermodynamic mixture of products in transfer hydrocyanation because both the linear and branched isomers are accessible. We surmised that in the absence of a Lewis acid co-catalyst a benzylic stabilization effect could lead to a markedly lower barrier for both the cleavage and formation of the branched nitrile product compared to the linear isomer, unlocking a branched selective transfer hydrocyanation of styrenes.

We verified this hypothesis experimentally through the reaction of 2-phenylpropionitrile $\mathbf{1}$ a with 4-tert-butylstyrene $\mathbf{2} \mathbf{b}$ and found that a mixture of the starting materials with styrene $\mathbf{2} \mathbf{a}$ and branched nitrile $\mathbf{1} \mathbf{b}$ was obtained while the linear isomers ( $\mathbf{3} \mathbf{a}$ and $\mathbf{3 b}$ ) were not observed (Scheme $2 \mathbf{a})$. This confirms that both the oxidative addition and reductive elimination can occur at the benzylic position in the absence of co-catalytic Lewis acid, whereas the formation of the linear product is considerably more challenging. Performing the reaction in reverse, gave an identical ratio of the two branched nitriles, a strong indicator that the reaction has reached equilibrium. The high barrier to oxidative addition of non-benzylic nitriles with the Lewis acid 
free system is also implicated by the lack of reactivity observed between 3-phenylpropionitrile 3a and 4-tert-butylstyrene $\mathbf{2 b}$ (Scheme $2 \mathbf{b}$ ). Furthermore, hydrocyanation of norbornadiene was not observed upon reaction with 2-phenylpropionitrile 1a (Scheme 2c). This truly underlines the challenging $\mathrm{C}-\mathrm{CN}$ reductive elimination at non-benzylic positions with this transfer hydrocyanation manifold, as norbornadiene is typically a potent acceptor for transfer reactions. ${ }^{6 \mathrm{~d}, 8 \mathrm{ce}}$ Collectively, these results show that, in the absence of co-catalytic Lewis acid, the reaction is still reversible and kinetically controlled toward selective formation and cleavage of the branched isomer, leaving other positions unreacted.

Next, we considered how to develop a practical, HCN-free transfer hydrocyanation process exhibiting such selectivity. While 2-phenylpropionitrile $1 \mathbf{a}$ is a competent $\mathrm{HCN}$ donor under the reaction conditions, it is not ideal for use in transfer hydrocyanation as an excess would be required to ensure good conversion of the substrate. Aside from being highly wasteful, this would also present problems with regards to purification and, therefore, an alternative $\mathrm{HCN}$ donor was devised. Desirable features include being able to donate $\mathrm{HCN}$ in the absence of a Lewis acid and it should also ideally form a stable alkene by-product that would be unsusceptible to hydrocyanation itself. We reasoned that malononitrile derivatives bearing an aliphatic group could fulfill these criteria while also being simply synthesized from inexpensive starting materials. ${ }^{16,17}$ It was anticipated that these species should be activated toward donating $\mathrm{HCN}$ to the Ni-catalyst ${ }^{18,19}$ and the conjugated alkenyl nitrile by-product should be recalcitrant to undergoing hydrocyanation. Gratifyingly, we discovered that malononitriles bearing a secondary aliphatic substituent $(\mathbf{4 a}-\mathbf{4 d})$ afforded high yields of product, although those bearing bulkier substituents (4e) failed in the reaction (Scheme 3). A simple isofunctional experiment confirmed that the dehydrocyanation of the reagent is irreversible under the reaction conditions. These results are also supported by comparison of the ground state free energies of reactants and products in the hydrocyanation of styrene $\mathbf{2 a}$ with malononitrile $\mathbf{4 a}$, which suggests that the reaction is distinctly exergonic $(\Delta G=-12.9 \mathrm{kcal} / \mathrm{mol}$ at $298 \mathrm{~K}$, see SI for details).

Scheme 2. Experiments to compare relative ease of $\mathrm{C}-\mathrm{CN}$ oxidative addition and reductive elimination at benzylic and non-benzylic positions.

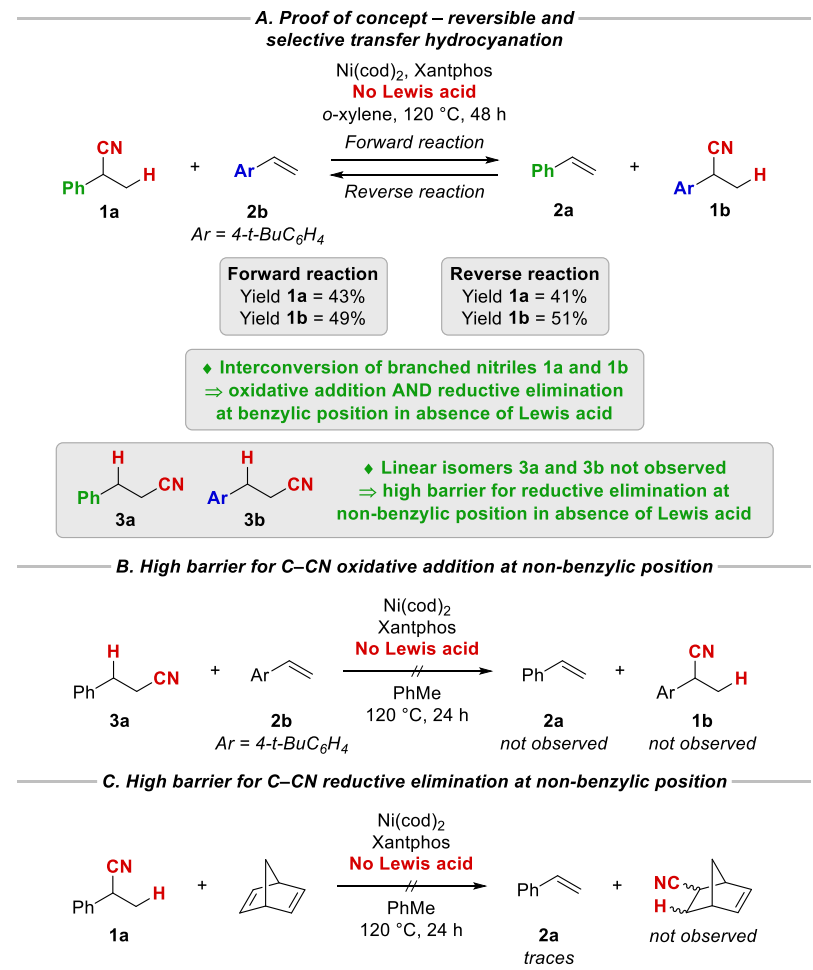


Scheme 3. Development of novel HCN donors for regioselective transfer hydrocyanation.

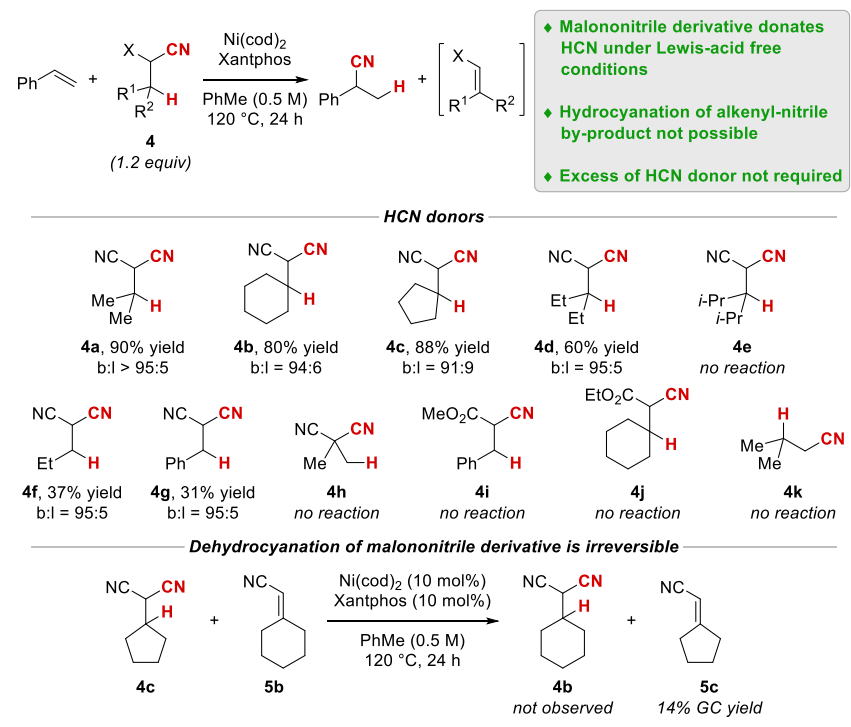

Following further optimization of the reaction conditions, the applicability of this $\mathrm{HCN}$-free, branched-selective transfer hydrocyanation was examined (Scheme 4). A variety of functional groups were well tolerated in the reaction including both electron donating and electron withdrawing moieties. The reaction also affords high yields and selectivities when performed with lower catalyst loadings (1o). Besides the high branched selectivity of this new reaction, which is complementary to previous protocols, we were also interested to see if it exhibits greater functional group tolerance, given that the current reaction conditions do not require co-catalytic Lewis acid. Indeed, substrates possessing acidic protons (1q-1v) or a Boc-protected amine $(\mathbf{1 u})$ were well tolerated. Finally, common heterocyclic cores $(\mathbf{1 w}-\mathbf{1 y})$ were found to successfully undergo transfer hydrocyanation with high selectivity. In cases with reduced yields, this was primarily due to low conversion.

Interestingly, while studying vinylheteroarenes as substrates in the reaction, a complete switch in regioselectivity was observed with 2-vinylpyridine $\mathbf{6}$ (Scheme 5). In this case, exquisite selectivity for the linear isomer 7 was observed. This is most likely due to a directing group effect, by the $\mathrm{N}$ atom of the pyridine, lowering the otherwise inaccessible energy barrier for $\mathrm{C}-\mathrm{CN}$ reductive elimination at non-benzylic positions. ${ }^{20}$ To further probe this coordinating effect, reaction of 6-methyl-2-vinylpyridine $\mathbf{8}$ was attempted and this was found to afford a mixture of both the linear (9) and branched (10) isomers, with the latter preferred. This lack of selectivity likely arises from the methyl group interfering with the ligation of the pyridine ring to the metal catalyst, allowing for the normal benzylic stabilization-controlled selectivity to compete with the directing group effect. In addition, linear isomer 7 could act as a HCN donor for hydrocyanation of simple styrenes (see SI for details), suggesting that the pyridine directing group also facilitates C$\mathrm{CN}$ oxidative addition at the non-benzylic position. Excitingly, even simple, unprotected alcohols (e.g. 11) afforded the linear product 12, suggesting that alcohols can also act as directing groups.

Scheme 4. Substrate scope for the regioselective, HCN-free transfer hydrocyanation., ${ }^{\mathrm{a}}$ 


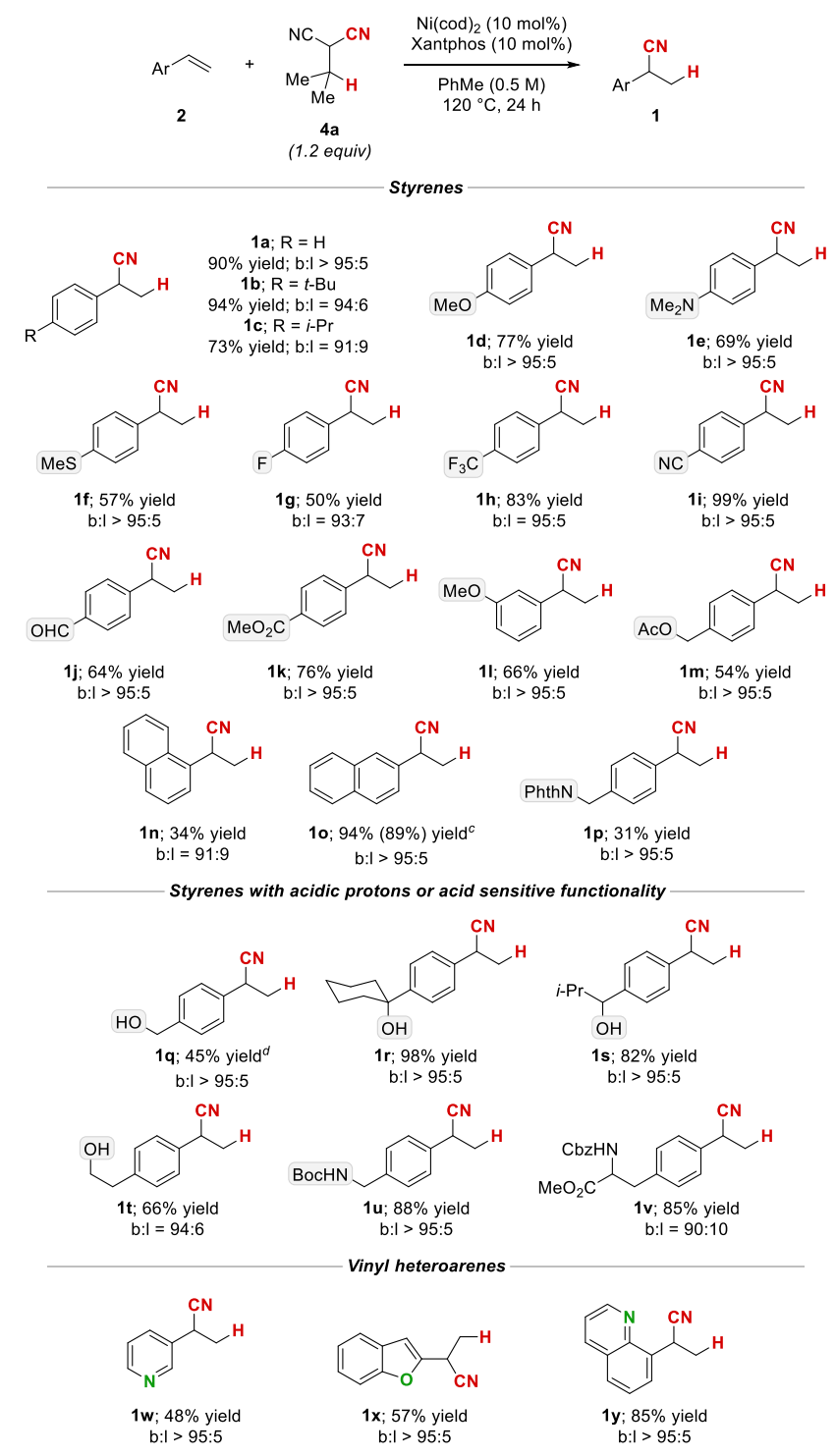

${ }^{a}$ Yields are given for isolated and purified material. Reactions were conducted on a $0.5 \mathrm{mmol} \mathrm{scale} .{ }^{b}$ Regioselectivity was determined by ${ }^{1} \mathrm{H}$ NMR analysis of the crude reaction mixture. 'Yield in parentheses for reaction performed on $2.0 \mathrm{mmol}$ scale with $2.5 \mathrm{~mol} \% \mathrm{Ni}(\operatorname{cod})_{2}$ and Xantphos. ${ }^{d} \mathrm{Aldehyde}$ $\mathbf{1 j}$ was also isolated in $33 \%$ yield.

Having found that directing groups could be used to control the selectivity of the reaction, we recognized that this provided an opportunity to explore less well-documented directing group effects. ${ }^{21}$ In particular, we were attracted to vinylsilanes as we reasoned that the silyl groups ability to stabilize both $\alpha$-anions and $\beta$-cations could provide another mechanism to lower the energy barrier for reductive elimination through inductive effects (Scheme 5). Accordingly, phenyldimethylvinylsilane 13 afforded the linear nitrile product $\mathbf{1 4}$ in high yields and selectivity, possibly hinting at the build-up of a positive charge at the $\beta$-position to the silicon during the $\mathrm{C}-\mathrm{CN}$ reductive elimination. 


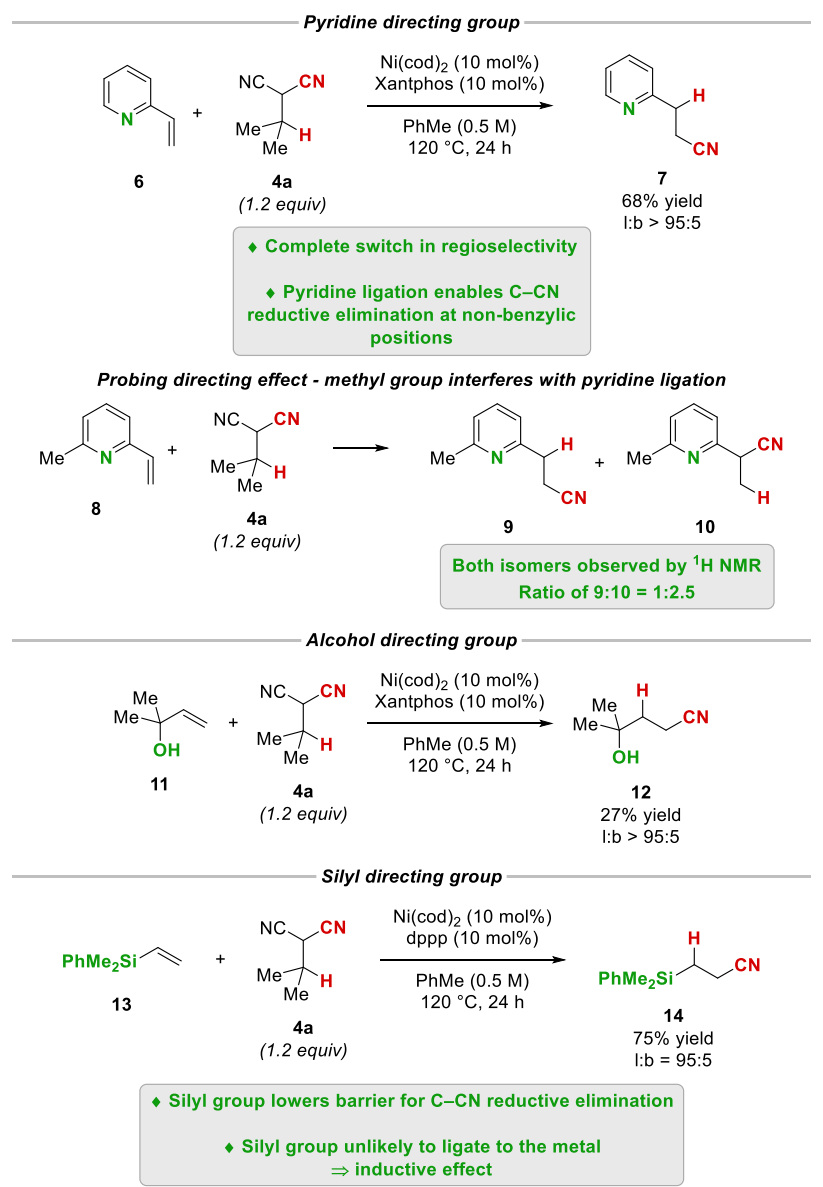

${ }^{a}$ Yields are given for isolated and purified material. Reactions were conducted on a $0.5 \mathrm{mmol}$ scale. ${ }^{b}$ Regioselectivity was determined by ${ }^{1} \mathrm{H}$ NMR analysis of the crude reaction mixture.

Finally, to further illustrate the value of this reversible and selective reaction, we used this new protocol to resolve the mixture of linear and branched isomers $(\mathrm{l}: \mathrm{b}=81: 19)$ obtained upon transfer hydrocyanation of styrene $2 \mathrm{a}$ using our previous, thermodynamically controlled protocol $($ Scheme 6$){ }^{8 \mathrm{~d}}$ By using an excess of commercially available 4-methoxystyrene, selective dehydrocyanation of the branched isomer was achieved while the linear isomer 3a could be recovered quantitatively with exquisite linear purity. In this manner, the linear nitrile can be obtained in high regiopurity through successive transfer hydrocyanations. This process is analogous to the resolution of a mixture of $E$ - and $Z$-alkenes by $Z$-selective ethenolysis to afford enrichment of the E-alkene content..$^{22}$

Preliminary deuterium labeling studies resulted in a statistical distribution of deuterium at both the branched and linear positions (Scheme 7 ). This suggests that a rapid pre-equilibrium has been reached and that the barrier for hydride insertion and $\beta$-hydride elimination is likely to be insignificant compared to the barrier for $\mathrm{C}-\mathrm{CN}$ reductive elimination. Thus, a rapid scrambling of the hydrogens at the branched and linear positions can occur. This result further verifies that selectivity in our reaction arises from the insurmountable barrier for $\mathrm{C}-\mathrm{CN}$ reductive elimination at the linear position in the absence of co-catalytic Lewis acid.

This observation, combined with the results shown in Schemes 2 and 3, allows us to understand the origins of the branched selectivity (Scheme 7). Irreversible dehydrocyanation of the malononitrile generates the corresponding $\mathrm{H}-\mathrm{Ni}-\mathrm{CN}$ intermediate. Rapid and reversible alkene insertion into the $\mathrm{Ni}-\mathrm{H}$ bond allows for an equilibrium to be established between the branched and the linear alkyl nickel intermediates. Finally, reversible reductive elimination to form the desired branched isomer occurs with high kinetic control over formation of the linear isomer.

\section{Scheme 6. Resolution of a regioisomeric mixture by selective dehydrocyanation.}




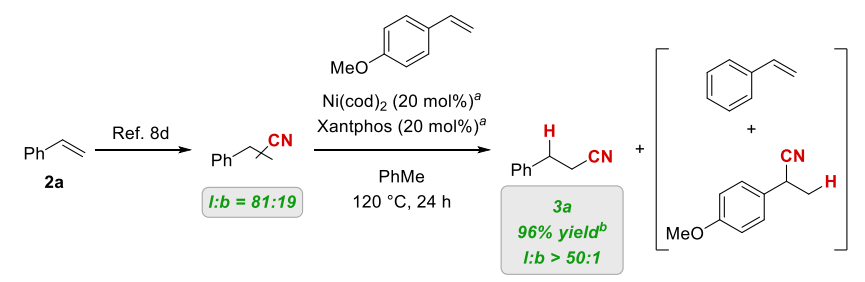

${ }^{a}$ Catalyst loadings are reported with respect to the amount of the branched isomer in the regioisomeric mixture. ${ }^{b}$ The yield was calculated by reference to an internal standard by GC-FID analysis and is given with respect to the amount of the linear isomer in the regioisomeric mixture.

\section{Scheme 7. Labeling studies and proposed catalytic cycle.}

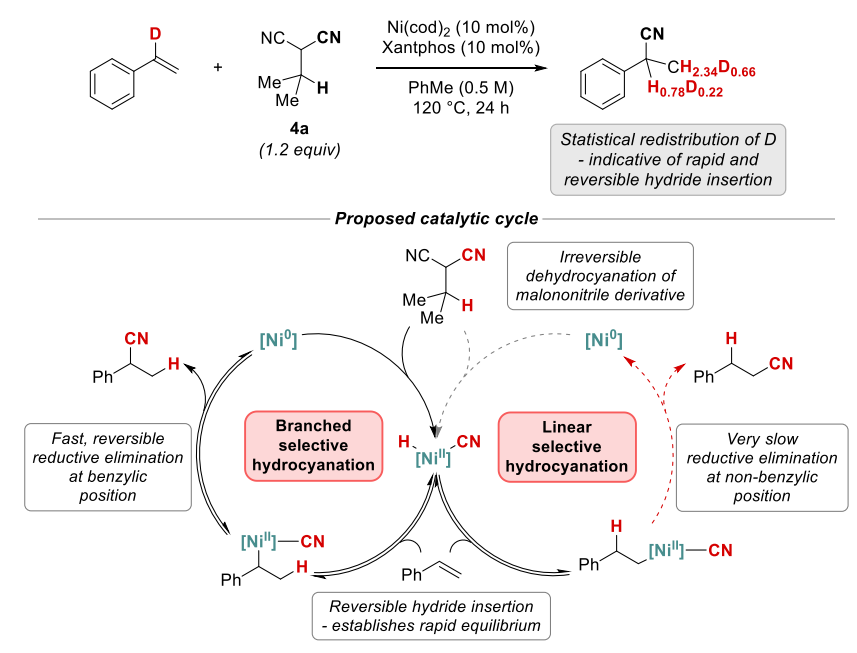

In conclusion, we have discovered a $\mathrm{HCN}$-free transfer hydrocyanation manifold that exhibits high kinetic selectivity for the thermodynamically less stable, branched isomer but, significantly, also remains reversible. Key to this selectivity is the lower barrier for $\mathrm{C}-\mathrm{CN}$ oxidative addition and reductive elimination at the benzylic position in the absence of co-catalytic Lewis acid. This manifold was exploited to achieve a highly selective transfer hydrocyanation of styrenes by using a novel design of $\mathrm{HCN}$ donor. To date, no other transfer reactions have been demonstrated to be both kinetically selective and reversible. Extending this concept could have enormous potential in organic synthesis, opening new strategies to both selectively functionalize multiple bonds and, through the reverse reaction, selectively defunctionalize complex molecules. 


\section{AUTHOR INFORMATION}

\section{Corresponding Author}

bill.morandi@org.chem.ethz.ch

\section{Notes}

A patent application for transfer hydrocyanation has been filed (US2019031602 (A1)).

\section{ACKNOWLEDGMENT}

This project received funding from the European Research Council under the European Union's Horizon 2020 research and innovation program (Shuttle Cat, Project ID: 757608). We also thank the Max-Planck-Society and ETH Zürich for support. We are also grateful to Prof. B. List for sharing analytical equipment. B.N.B. acknowledges the Leverhulme Trust and the ERC for funding. J.C.R. acknowledges a fellowship of the Stipendienfonds Schweizerische Chemische Industrie (SSCI). We thank Prof. Z. K. Wickens (UW-Madison) and Prof. R. H. Grubbs (CalTech) for helpful discussions.

\section{REFERENCES}

(1) (a) Grubbs, R. H. Handbook of Metathesis (Wiley-VCH, Weinheim, Germany, 2003). (b) Fürstner, A. Olefin metathesis and beyond. Angew. Chem. Int. Ed. 2000, 39, 3012. (c) Schrock, R. R.; Hoveyda, A. H. Molybdenum and Tungsten Imido Alkylidene Complexes as Efficient Olefin-Metathesis Catalysts. Angew. Chem. Int. Ed. 2003, 42, 4592. (d) Chatterjee, A. K.; Choi, T.-L.; Sanders, D. P.; Grubbs, R. H. A General Model for Selectivity in Olefin Cross Metathesis. J. Am. Chem. Soc. 2003, 125, 11360 .

(2) (a) Brieger, G.; Nestrick, T.J. Catalytic Transfer Hydrogenation. Chem. Rev. 1974, 74, 567. (b) Wang, D.; Astruc, D. The Golden Age of Transfer Hydrogenation. Chem. Rev. 2015, 115,6621

(3) For examples of the use of metathesis in total synthesis, see: (a) Nicolaou, K. C.; Bulger, P. G.; Sarlah, D. Metathesis Reactions in Total Synthesis. Angew. Chem. Int. Ed. 2005, 44, 4490. (b) Fürstner, A. Metathesis in Total Synthesis. Chem. Commun. 201 1, 47, 6505.

(4) For examples of the use of alkene metathesis in polymerization, see: Ivin, K.; Mol, H. Olefin Metathesis and Metathesis Polymerization (Elsevier, Amsterdam, 1997).

(5) For examples of the use of transfer hydrogenation in borrowing hydrogen, see: (a) Hamid, M. H. S. A.; Slatford, P. A.; Williams, J. M. J. Borrowing Hydrogen in the Activation of Alcohols. Adv. Synth. Catal. 2007, 349, 1555. (b) Bower, J. F.; Kim, I. S.; Patman, R. L.; Krische, M. J. Catalytic Carbonyl Addition through Transfer Hydrogenation: A Departure from Preformed Organometallic Reagents. Angew. Chem. Int. Ed. 2009, 48, 34. (c) Dobereiner, G. E.; Crabtree, R. H. Dehydrogenation as a Substrate-Activating Strategy in Homogeneous Transition-Metal Catalysis. Chem. Rev. 2010, 110, 681. (d) Guillena, G.; Ramón, D. J.; Yus, M. Hydrogen Autotransfer in the N-Alkylation of Amines and Related Compounds using Alcohols and Amines as Electrophiles. Chem. Rev. 2010, 110, 1611. (e) Corma, A.; Navas, J.; Sabater, M. J. Advances in One-Pot Synthesis through Borrowing Hydrogen Catalysis. Chem. Rev. 2018, 118, 1410.

(6) For reviews, see: (a) Bhawal, B.N.; Morandi, B. Catalytic Isofunctional Reactions-Expanding the Repertoire of Shuttle and Metathesis Reactions. Angew. Chem. Int. Ed. 2019, 58, 10074. (b) Becker, M.R.; Watson, R. B.; Schindler, C. S. Beyond Olefins: New Metathesis Directions for Synthesis. Chem. Soc. Rev. 2018, 47, 7867. (c) Bhawal, B. N.; Morandi, B. Isodesmic Reactions in Catalysis - Only the Beginning? Isr. J. Chem. 2018, 58, 94. (d) Bhawal, B. N.; Morandi, B. Catalytic Transfer Functionalization through Shuttle Catalysis. ACS Catal. 2016, 6, 7528.

(7) For representative examples of metathesis reactions, see: (a) Goldman, A. S.; Roy, A. H.; Huang, Z.; Ahuja, R.; Schinski, W.; Brookhart, M. Catalytic Alkane Metathesis by Tandem Alkane Dehydrogenation-Olefin Metathesis. Science 2006, 312, 257. (b) Geyer, A. M.; Gdula, R. L.; Wiedner, E. S.; Johnson, M. J. A. Catalytic Nitrile-Alkyne Cross-Metathesis. J. Am. Chem. Soc. 2007, 129, 3800. (c) Ludwig, J. R.; Zimmerman, P. M.; Gianino, J. B.; Schindler, C. S. Iron(III)-Catalysed CarbonylOlefin Metathesis. Nature 2016, 533, 374. (d) Lian, Z.; Bhawal, B. N.; Yu, P.; Morandi, B. Palladium-Catalyzed Carbon-Sulfur or Carbon-Phosphorus Bond Metathesis by Reversible Arylation. Science 2017, 356, 1059. (e) Biberger, T.; Makai. S.; Lian, Z.; Morandi, B. Iron-Catalyzed Ring-Closing C-O/C-O Metathesis of Aliphatic Ethers. Angew. Chem. Int. Ed. 2018, 57, 6940. (f) Lee, Y. H.; Morandi, B. Metathesis-Active Ligands Enable a Catalytic Functional Group Metathesis Between Aroyl Chlorides and Aryl Iodides. Nat. Chem. 2018, 10, 1016. (g) Macias, M. D. L. H.; Arndtsen, B. A. Functional Group Transposition: A Palladium-Catalyzed Metathesis of Ar-X oBonds and Acid Chloride Synthesis. J. Am. Chem. Soc. 2018, 140, 10140.

(8) For representative examples of isodesmic transfer reactions, see: (a) Jun, C.-H.; Lee, H. Catalytic Carbon-Carbon Bond Activation of Unstrained Ketone by Soluble Transition-Metal Complex. J. Am. Chem. Soc. 1999, 121, 880. (b) Greenhalgh, M. D.; Thomas, S. P. Iron-Catalyzed, Highly Regioselective Synthesis of $a$-Aryl Carboxylic Acids from Styrene Derivatives and CO2.J.Am. Chem. Soc. 2012, 134, 11900. (c) Murphy, S. K.; Park, J.-W.; Cruz, F.A.; Dong, V.M. Rh-Catalyzed C-C Bond Cleavage by Transfer Hydroformylation. Science 2015, 347, 56. (d) Fang, X.; Yu, P.; Morandi, B. Catalytic Reversible Alkene-Nitrile Interconversion Through Controllable Transfer Hydrocyanation. Science 2016, 351, 832. (e) Fang, X.; Cacherat, B.; Morandi, B. CO- and HCl-free Synthesis of Acid Chlorides from Unsaturated Hydrocarbons via Shuttle Catalysis. Nat. Chem. 2017, 9, 1105.

(9) (a) Bhunia, A.; Bergander, K.; Studer, A. Cooperative Palladium/Lewis Acid-Catalyzed Transfer Hydrocyanation of Alkenes and Alkynes Using 1-Methylcyclohexa-2,5-diene-1-carbonitrile. J. Am. Chem. Soc. 2018, 140, 16353. (b) Ye, F.; Chen, J.; Ritter, T. Rh-Catalyzed Anti-Markovnikov Hydrocyanation of Terminal Alkynes. J. Am. Chem. Soc. 2017, 139, 7184. (c) Zhang, X.; Xie, X.; Liu, Y. Nickel-Catalyzed Highly Regioselective Hydrocyanation of Terminal Alkynes with Zn(CN) 2 Using Water as the Hydrogen Source. J. Am. Chem. Soc. 2018, 140, 7385. (d) Schluppe, A. W.; Borrajo-Calleja, G. M.; Buchwald, S. L. Enantioselective Olefin Hydrocyanation without Cyanide. J. Am. Chem. Soc. 2019, 141, 18668. (e) Orecchia, P.; Yuan, W.; Oestreich, M. Transfer Hydrocyanation of $\alpha$ - and $\alpha$, $\beta$-Substituted Styrenes Catalyzed by Boron Lewis Acids. Angew. Chem. Int. Ed. 2019, 58, 3579. (f) Yang, L.; Liu, Y.-T.; Park, Y.; Park, S.-W.; Chang, S. Ni-Mediated Generation of "CN" Unit from Formamide and Its Catalysis in the Cyanation Reactions. ACS Catal. 2019, 9, 3360. (g) Jia, T.; Smith, M. J.; Pulis, A. P.; Perry, G. J. P.; Procter, D. J. Enantioselective and Regioselective Copper-Catalyzed Borocyanation of 1-Aryl-1,3-Butadienes. ACS Catal. 2019, 9, 6744. (h) Wang, G.; Xie, X.; Xu, W.; Liu, Y. Nickel-Catalyzed Highly Regioselective Hydrocyanation of Alkenes with $\mathrm{Zn}(\mathrm{CN})_{2}$. Org. Chem. Front. 2019, 6, 2037. For other examples of hydrocyanation, see: (i) Gaspar, B.; Carreira, E. M. Mild Cobalt-Catalyzed Hydrocyanation of Olefins with Tosyl Cyanide. Angew. Chem. Int.Ed. 2007, 46, 4519. (j) Arai, S.; Hori, H.; Amako, Y.; Nishida, A. A New Protocol for Nickel-Catalysed Regio- and Stereoselective Hydrocyanation of Allenes. Chem. Commun. 2015, 51, 7493. (k) Kristensen, S. K; Eikeland, E.Z.; Taarning, E.; Lindhardt, A. T.; Skrydstrup, T. Ex situ generation of stoichiometric HCN and its application in the Pd-catalysed cyanation of aryl bromides: evidence for a transmetallation step between two oxidative addition Pd-complexes. Chem. Sci. 2017, 8, 8094.

(10) For a review, see: Kim, J.; Kim, H. J.; Chang, S. Synthesis of Aromatic Nitriles Using Nonmetallic Cyano-Group Sources. Angew. Chem. Int. Ed. 2012, 51 , 11948.

(11) For selected examples, see: (a) Kim, J.; Chang, S. A New Combined Source of "CN" from N,N-Dimethylformamide and Ammonia in the Palladium-Catalyzed Cyanation of Aryl C-H Bonds. J. Am. Chem. Soc. 2010, 132, 10272. (b) Kim, J.; Choi, J.; Shin, K.; Chang, S. Copper-Mediated Sequential Cyanation of Aryl C-B and Arene C-H Bonds Using Ammonium Iodide and DMF. J. Am. Chem. Soc. 2012, 134, 2528. (c) Pawar, A. B.; Chang, S. Catalytic Cyanation of Aryl Iodides Using DMF 
and Ammonium Bicarbonate as the Combined Source of Cyanide: a Dual Role of Copper Catalysts. Chem. Commun. 2014, 50, 448. (d) Senecal, T.D.; Shu, W.; Buchwald, S. L. A General, Practical Palladium-Catalyzed Cyanation of (Hetero)Aryl Chlorides and Bromides. Angew. Chem. Int. Ed. 2013, 52, 10035. (e) Yang, Y.; Buchwald, S. L. Copper-Catalyzed Regioselective ortho C-H Cyanation of Vinylarenes. Angew. Chem. Int. Ed. 2014, 53, 8677. (f) Yu, P.; Morandi, B. Nickel-Catalyzed Cyanation of Aryl Chlorides and Triflates Using Butyronitrile: Merging Retro-Hydrocyanation with Cross-coupling. Angew. Chem. Int. Ed. 2017, 56, 15693. (g) Huang, Y.; Yu, Y.; Zhu, Z.; Zhu, C.; Cen, J.; Li, X.; Wu, W.; Jiang, H. Copper-Catalyzed Cyanation of N-Tosylhydrazones with Thiocyanate Salt as the “CN” Source. J. Org. Chem. 2017, 82, 7621. (h) Michel, N. W. M.; Jeanneret, A.D. M.; Kim, H.; Rousseaux, S. A. L. Nickel-Catalyzed Cyanation of Benzylic and Allylic Pivalate Esters. J. Org. Chem. 2018, 83, 11860. (i) Ueda, Y.; Tsujimoto, N.; Yurino, T.; Tsurugi, H.; Mashima, K. Nickel-Catalyzed Cyanation of Aryl Halides and Triflates Using Acetonitrile via C-CN Bond Cleavage Assisted by 1,4-bis(trimethylsilyl)-2,3,5,6-tetramethyl-1,4-dihydropyrazine. Chem. Sci. 2019, 10, 994. (j) Holmberg-Douglas, N.; Nicewicz, D. A. Arene Cyanation via Cation-Radical Accelerated-Nucleophilic Aromatic Substitution. Org. Lett. 2019, 21, 7114. (k) Xu, S.; Teng, J.; Yu, J.-T.; Sun, S.; Cheng, J. Copper-Mediated Direct Cyanation of Heteroarene and Arene C-H Bonds by the Combination of Ammonium and DMF. Org. Lett. 2019, 21, 9919. (1) Chen, H.; Mondal, A.; Wedi, P.; van Gemmeren, M. Dual Ligand-Enabled Nondirected C-H Cyanation of Arenes. ACS Catal. 2019, 9, 1979.

(12) The relative gas-phase free energies for 2-phenylpropionitrile and 3-phenylpropionitrile were calculated (see SI for details). It was found that the lowest energy conformer of the linear isomer, 3-phenylpropionitrile, is more stable than the lowest energy conformer of the branched isomer, 2-phenylpropionitrile, by $1.3 \mathrm{kcal} / \mathrm{mol}$.

(13) (a) Flook, M. M.; Jiang, A.J.; Schrock, R. R.; Müller, P.; Hoveyda, A. H.Z-Selective Olefin Metathesis Processes Catalyzed by a Molybdenum Hexaisopropylterphenoxide Monopyrrolide Complex. J. Am. Chem. Soc. 2009, 131, 7962. (b) Jiang, A. J.; Zhao, Y.; Schrock, R. R.; Hoveyda, A. H. Highly Z-Selective Metathesis Homocoupling of Terminal Olefins. J. Am. Chem. Soc. 2009, 131, 16630. (c) Yu, M.; Wang, C.; Kyle, A. F.; Jakubec, P.; Dixon, D. J.; Schrock, R. R.; Hoveyda, A. H. Synthesis of Macrocyclic Natural Products by Catalyst-Controlled Stereoselective Ring-Closing Metathesis. Nature 201 1, 479, 88. (d) Meek, S. J.; O’Brien, R. V.; Llaveria, J.; Schrock, R. R; Hoveyda, A. H. Catalytic Z-selective Olefin Cross-Metathesis for Natural Product Synthesis. Nature 2011, 471, 461. (e) Endo, K.; Grubbs, R. H. Chelated Ruthenium Catalysts for Z-Selective Olefin Metathesis. J. Am. Chem. Soc. 2011, 133, 8525. (f) Keitz, B. K.; Endo, K.; Herbert, M. B.; Grubbs, R. H. Z-Selective Homodimerization of Terminal Olefins with a Ruthenium Metathesis Catalyst. J. Am. Chem. Soc. 2011, 133, 9686. (g) Marx, V. M.; Herbert, M. B.; Keitz, B. K.; Grubbs, R. H. Stereoselective Access to $Z$ and $E$ Macrocycles by Ruthenium-Catalyzed Z-Selective Ring-Closing Metathesis and Ethenolysis. J. Am. Chem. Soc. 2013, 135, 94. (h) Hoveyda, A. H. Evolution of Catalytic Stereoselective Olefin Metathesis: From Ancillary Transformation to Purveyor of Stereochemical Identity. J. Org. Chem. 2014, 79, 4763. (i) Herbert, M. B.; Grubbs, R. H. Z-Selective Cross Metathesis with Ruthenium Catalysts: Synthetic Applications and Mechanistic Implications. Angew. Chem. Int. Ed. 2015, $54,5018$.

(14) (a) Tolman, C. A.; Seidel, W.C.; Druliner, J.D.; Domaille, P.J. Catalytic Hydrocyanation of Olefins by Nickel(0) Phosphite Complexes - Effects of Lewis Acids. Organometallics 1984, 3, 33. (b) Casalnuovo, A. L.; RajanBabu, T. V.; Ayers, T. A.; Warren, T. H. Ligand Electronic Effects in Asymmetric Catalysis: Enhanced Enantioselectivity in the Asymmetric Hydrocyanation of Vinylarenes.J. Am. Chem. Soc. 1994, 116, 9869. (c) RajanBabu, T. V.; Casalnuovo, A. L. Role of Electronic Asymmetry in the Design of New Ligands: The Asymmetric Hydrocyanation Reaction. J. Am. Chem. Soc. 1996, 118, 6325. (d) Huang, J.; Haar, C.M.; Nolan, S.P.; Marcone, J.E.; Moloy, K.G. Lewis Acids Accelerate Reductive Elimination of RCN from P2Pd(R)(CN). Organometallics 1999, 18, 297. (e) Nakao, Y.; Yada, A.; Ebata, S.; Hiyama, T. A Dramatic Effect of Lewis-Acid Catalysts on Nickel-Catalyzed Carbocyanation of Alkynes. J. Am. Chem. Soc. 2007, 129, 2428. (f) Bini, L.; Müller, C.; Vogt, D. Mechanistic Studies on Hydrocyanation Reactions. Chem CatChem 2010, 2, 590. (g) Rajanbabu, T. V. Hydrocyanation of Alkenes and Alkynes. Org. React. 2011, 75, 1. (h) Ni, S.-F.; Yang, T.-L.; Dang, L. Transfer Hydrocyanation by Nickel(0)/Lewis Acid Cooperative Catalysis, Mechanism Investigation, and Computational Prediction of Shuttle Catalysts. Organometallics 2017, 36, 2746.

(15) For a review on the role of Lewis acids in transition metal catalysis, see: Becica, J.; Dobereiner, G.E. The roles of Lewis acidic additives in organotransition metal catalysis. Org. Biomol. Chem. 2019, 17, 2055.

(16) Sammelson, R. E.; Allen, M. J. A Convenient and Selective One-Pot Method for the Synthesis of Monosubstituted Secondary Alkyl Malononitriles. Synthesis 2005, 543 .

(17) While malononitrile, from which the HCN donors 4a-g are synthesized, is easier to handle than $\mathrm{HCN}$ care should be taken when using malononitrile as it is toxic. See: Malononitrile; MSDS No. M1407; Sigma-Aldrich Company: Gillingham, Dorset, May 07, 2020.

(18) For examples of C-X oxidative addition adjacent to nitriles, see: (a) Cannes, C.; Condon, S.; Durandetti, M.; Périchon, J.; Nédélec, J.-Y. Nickel-Catalyzed Electrochemical Couplings of Vinyl Halides: Synthetic and Stereochemical Aspects. J. Org. Chem. 2000, 65, 4575. (b) Strotman, N. A.; Sommer, S.; Fu, G. C. Hiyama Reactions of Activated and Unactivated Secondary Alkyl Halides Catalyzed by a Nickel/Norephedrine Complex. Angew. Chem. Int. Ed. 2007, 46, 3556. (c) He, A.; Falck, J. R. Stereospecific Suzuki Cross-Coupling of Alkyl $\alpha$-Cyanohydrin Triflates. J. Am. Chem. Soc. 2010, 132, 2524. (d) Yang, Y.; Tang, S.; Liu, C.; Zhang, H.; Sun, Z.; Lei, A. Novel $\alpha$-Arylnitriles Synthesis via Ni-Catalyzed Cross-Coupling of $\alpha$-Bromonitriles with Arylboronic Acids Under Mild Conditions. Org. Biomol. Chem. 2011, 9, 5343. (e) Choi, J.; Fu, G. C. Catalytic Asymmetric Synthesis of Secondary Nitriles via Stereoconvergent Negishi Arylations and Alkenylations of Racemic $\alpha$-Bromonitriles. J. Am. Chem. Soc. 2012, 134, 9102. (f) Tang, S.; Liu, C.; Lei, A. Nickel-Catalysed Novel $\beta, \gamma$-unsaturated Nitrile Synthesis. Chem. Commun. 2013, 49, 2442. (g) Kadunce, N. T. Reisman, S. E. Nickel-Catalyzed Asymmetric Reductive Cross-Coupling between Heteroaryl Iodides and $\alpha$-Chloronitriles. J. Am. Chem. Soc. 2015, 137, 10480. (h) Donslund, A. S.; Neumann, K. T.; Corneliussen, N. P.; Grove, E. K.; Herbstritt, D.; Dassbjerg, K.; Skrydstrup, T. Access to $\beta$-Ketonitriles through Nickel-Catalyzed Carbonylative Coupling of $\alpha$-Bromonitriles with Alkylzinc Reagents. Chem. Eur. J. 2019, 25, 9856. For examples of C-CN oxidative addition, see: (i) Nakao, Y.; Oda, S.; Hiyama, T. Nickel-Catalyzed Arylcyanation of Alkynes. J. Am. Chem. Soc. 2004, 126, 13904.

(19) Recently, disubstituted malononitrile derivatives have been used in the cyanation of aryl (pseudo)halides, proceeding through a transnitrilation mechanism. (a) Reeves, J. T.; Malapit, C. A.; Buono, F. G.; Sidhu, K. P.; Marsini, M. A.; Sader, C. A.; Fandrick, K. R.; Busacca, C. A.; Senanayake, C.H. Transnitrilation from Dimethylmalononitrile to Aryl Grignard and Lithium Reagents: A Practical Method for Aryl Nitrile Synthesis. J. Am. Chem. Soc. 2015, 137, 9481. (b) Malapit, C. A.; Reeves, J. T.; Busacca, C. A.; Howell, A. R.; Senanayake, C. H. Rhodium-Catalyzed Transnitrilation of Aryl Boronic Acids with Dimethylmalononitrile. Angew. Chem. Int. Ed. 2016, 55, 326. (c) Alazet, S.; West, M. S.; Patel, P.; Rousseaux, S. A. L. Synthesis of Nitrile-Bearing Quaternary Centers by an Equilibrium-Driven Transnitrilation and AnionRelay Strategy. Angew. Chem. Int. Ed. 2019, 58, 10300. (d) Mills, L. R.; Graham, J. M.; Patel, P.; Rousseaux, S. A. L. Ni-Catalyzed Reductive Cyanation of Aryl Halides and Phenol Derivatives via Transnitrilation.J.Am. Chem. Soc. 2019, 141, 19257.

(20) For an example of directing group effects in carbocyanation, see: (a) Nakao, Y.; Yada, A.; Hiyama, T. Heteroatom-Directed Alkylcyanation of Alkynes. J. Am. Chem. Soc. 2010, 132, 10024. For reviews on directing group effects, see: (b) Hoveyda, A. H.; Evans, D. A.; Fu, G. C. Substrate-directable chemical reactions. Chem. Rev. 1993, 93, 1307. (c) Lyons, T. W.; Sanford, M. S. Palladium-Catalyzed Ligand-Directed C-H Functionalization Reactions. Chem. Rev. 2010, 110, 1147. (d) Rousseau, G.; Breit, B. Removable Directing Groups in Organic Synthesis and Catalysis. Angew. Chem. Int. Ed. 2011, 50, 2450. (e) Sambiagio, C.; Schönbauer, D.; Blieck, R.; Dao-Huy, T.; Pototschnig, G.; Schaaf, P.; Wiesinger, T.; Zia, M. F.; Wencel-Delord, J.; Besset, T.; Maes, B. U. W.; Schnürch, M. A Comprehensive Overview of Directing Groups Applied in Metal-Catalysed C-H Functionalisation Chemistry. Chem. Soc. Rev. 2018, 47, 6603.

(21) Haines, B. E.; Sarpong, R.; Musaev, D. G. Generality and Strength of Transition Metal $\beta$-Effects. J. Am. Chem. Soc. 2018, 140, 10612.

(22) (a) Marinescu, S. C.; Levine, D. S.; Zhao, Y.; Schrock, R. R.; Hoveyda, A. H. Isolation of Pure Disubstituted E Olefins through Mo-Catalyzed Z-Selective Ethenolysis of Stereoisomeric Mixtures. J. Am. Chem. Soc. 2011, 133, 11512. (b) Marx, V. M.; Herbert, M. B.; Keitz, B. K.; Grubbs, R. H. Stereoselective Access to Z and E Macrocycles by Ruthenium-Catalyzed Z-Selective Ring-Closing Metathesis and Ethenolysis. J. Am. Chem. Soc. 2013, 135, 94. (c) Miyazaki, H.; Herbert, M. B.; Liu, P.; 
Dong, X.; Xu, X.; Keitz, B. K.; Ung, T.; Mkrtumyan, G.; Houk, K. N.; Grubbs, R. H. Z-Selective Ethenolysis with a Ruthenium Metathesis Catalyst: Experiment and Theory. J. Am. Chem. Soc. 2013, 135, 5848. 


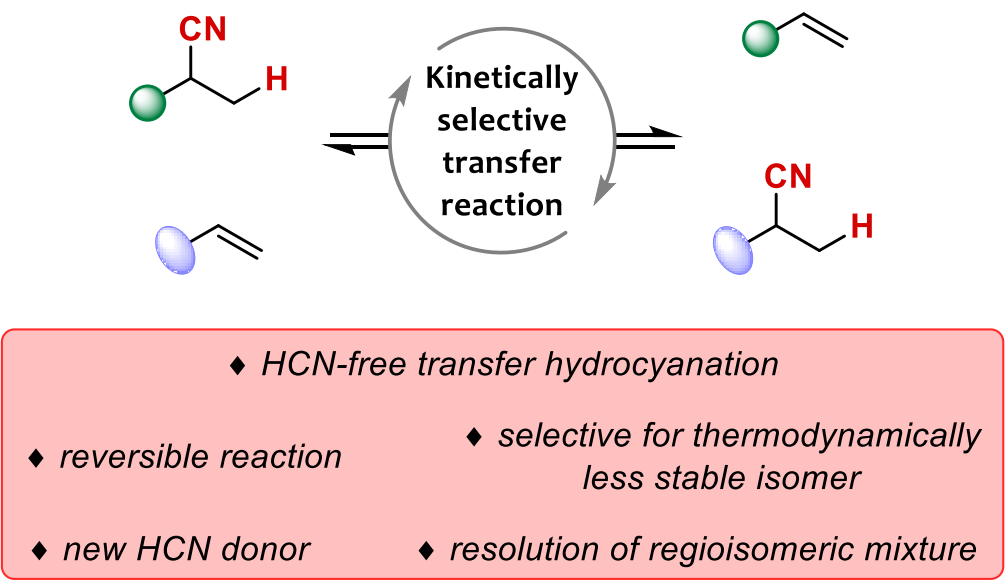

